\title{
New mechanisms for international coordination of large observing projects
}

\author{
Johannes Andersen \\ The Niels Bohr Institute, University of Copenhagen \\ and Nordic Optical Telescope, La Palma, Spain \\ Juliane Maries Vej 30, DK-2100 Copenhagen, Denmark \\ E-mail: ja@astro.ku.dk
}

\begin{abstract}
Progress on many fronts in the study of the Milky Way requires major new imaging and/or spectroscopic surveys as well as coordinated ground-based programmes in support of space missions. The present uncoordinated planning and operation of the $2-4 \mathrm{~m}$ (and some of the larger) telescopes across the world are an impediment to the efficient conduct of such programmes and the overall cost-effectiveness of the investments in astronomy. We briefly report on recent initiatives to improve the situation, notably the part played by the ASTRONET consortium in setting up a comprehensive long-term plan for the development of European astronomy.
\end{abstract}

Keywords. sociology of astronomy

\section{Background}

As shown in many contributions to this session, progress on several fronts in our understanding of the origin and evolution of disk galaxies require homogeneous high-quality data for ever larger samples of objects - be they galaxies in the distant Universe or individual stars or gas clouds in the Milky Way and its neighbours. Some such programmes are self-contained ground- or space-based projects, but many involve a combination of observations from space and from the ground.

In practical terms, such projects typically require hundreds of nights on 2-4m-class ground-based telescopes, with optimised instrumentation, data reduction pipelines, and the staff to run it all efficiently. As a rule, coordination is needed, either to ensure spectroscopic coverage of objects selected in imaging surveys; to ensure that the spaceand ground-based data are obtained and combined to reach the scientific objectives in a timely manner; or simply to achieve all-sky coverage.

The scientific advantages of such coordinated programmes are obvious. Yet, their practical implementation is impeded by the way our telescopes are traditionally organised, operated and funded: On an institutional, national or at best regional basis (e.g. ESO). Observing time is allocated to individual P.I.s, based on proposals ranked by individual scientific merit rather than by the global scientific returns of the national investments, and with no overall coordination of instrumentation or scheduling.

Coordination of ground-based follow-up with space missions has been especially weak in Europe. As just one example, the Geneva-Copenhagen Survey of the Solar Neighbourhood (Nordström et al. 2004) provided photometry and radial velocities for $\sim 15 \%$ of the stars observed by Hipparcos, several years after the astrometry was complete and after 20 years of struggle by a small team with private telescopes. Complete, homogeneous ground-based data for all the stars could have been ready in time for $\sim 2 \%$ of the cost of the Hipparcos mission itself, but rigid policies prevented this from happening, at great loss in scientific returns from the investment in the satellite. 
Conversely, the Sloan Survey owes its success to the coherent planning of the imaging and spectroscopic parts of the survey, with a dedicated telescope and the resources needed to provide the processed data in a timely manner. Yet, the Sloan Survey is limited to the northern hemisphere, just as the RAVE survey is currently limited to the southern hemisphere for lack of a suitable northern telescope with the required funding. The scientific drawbacks of this situation are obvious.

\section{A new start - hopefully!}

Recent initiatives have been taken to improve this situation. Planning exercises both in the USA and Europe are aiming to review the role and organisation of medium-size ground-based telescopes as part of the overall complement of facilities needed to reach overarching scientific goals.

In the USA, the NOAO has appointed the ReSTAR and ALTAIR committees to review the future role and organisation of the US system of $2-4 \mathrm{~m}$ and $6-10 \mathrm{~m}$ telescopes, respectively. In Europe, similar reviews are part of the recent, comprehensive ASTRONET planning initiative. Because ASTRONET is probably not yet familiar to most of the community, a short account is provided here.

ASTRONET is a consortium of national funding agencies for astronomy in Europe, plus the European Southern Observatory (ESO) and the European Space Agency (ESA). It was founded in 2005 and is supported by the European Commission with a four-year, 2.5 MEuro grant under the ERA-NET scheme. Its goal is to develop a long-term plan for the science-based, rational, and coordinated development of European astronomy somewhat akin to the Decadal Surveys in the USA, but with significant differences.

Notably, the science drivers for the plan were first collected, prioritised, and published as a separate exercise. The report, A Science Vision for European Astronomy (de Zeeuw $\&$ Molster 2007), focused the discussion on four broad questions:

Do we understand the extremes of the Universe?

How do galaxies form and evolve?

How do stars and planets form? and

How do we fit in?

Extensive community input on a first draft of the Science Vision was collected at a large open Symposium and via a web-based forum both before and after the Symposium.

With the Science Vision as the scientific basis, a comprehensive Infrastructure Roadmap was then developed (Bode et al. 2008) to describe the resources needed to reach these scientific goals. The Roadmap is also structured according to a few major headings:

High energy astrophysics, astroparticles and gravitational waves, UV, optical, IR and radio/mm facilities, including survey instruments,

Solar telescopes and "in situ" Solar System missions,

Theory, computing facilities, networks, Virtual Observatory, and

Education, recruitment and training, and public outreach.

The Roadmap lists and prioritises the major new research facilities required - at all wavelengths of the electromagnetic spectrum as well as for particles and gravitational waves - and in space as well as on the ground. It also includes the concomitant investments in laboratory astrophysics and in theory, computing, networks, and archiving, including the Virtual Observatory. 
Last, but not least, it also assesses the measures needed to provide the necessary skilled humans - education, recruitment, and outreach - and it includes not only the established ESO and ESA member states, but all of Europe as we know it today. As for the Science Vision, community input was actively involved in its preparation.

The Roadmap was developed in close cooperation with the ERA-NET ASPERA for astroparticle physics, and the infrastructure coordination networks OPTICON, RadioNet, ILIAS, and EuroPlaNet, which support the hands-on implementation of European cooperation on the facilities in their respective domains. Close contact to the funding agencies was also maintained to ensure that realistic costs of construction, operation, and exploitation were included in the budget estimates, and that the overall cost and spending profiles remained in touch with reality.

In parallel, ASTRONET worked to include all astronomical communities in its endeavour. Currently, 25 Member and Associated States of the European Union, with a combined population of over 500 million people, are associated with ASTRONET in some form or other.

The publication of the Roadmap immediately marks the start of the implementation phase, and initiatives are already being taken to carry out its recommendations. To prepare the future decisions on the largest infrastructure projects, contacts are being taken to the European Strategy Forum for Research Infrastructures (ESFRI) and the OECD Global Science Forum (GSF). As regards the smaller, existing facilities, action is being taken by ASTRONET itself, as described below.

\section{Coordination of existing telescope facilities}

In order to review the existing European complement of 2-4m telescopes and propose innovative measures to organise them in a more cost-effective manner, the ASTRONET Board appointed a European Telescope Strategy Review Committee in June 2008. Its report is due in the autumn of 2009 and will address a number of key questions:

- Identify those goals of the ASTRONET Science Vision that are most effectively delivered by $2-4 \mathrm{~m}$-class optical/infrared telescopes;

- Identify which observational capabilities (site, field of view, instrumentation, modes of operation) are required; and

- Develop a realistic roadmap, including technical developments and upgrades, and organisational/financial arrangements, which would enable a set of European 2-4m-class telescopes to deliver the best scientific output for European astronomy in a cost-effective manner.

The benefits expected to accrue from this exercise include:

Ability to implement large (all-sky) programmes (e.g. RAVE+)

Ability to implement coordinated ground/space projects (e.g. Gaia)

Include new partners, new and more flexible funding sources, and

More effective coordination with world-wide partners.

In short, the goal is to do more and better science through more intelligent international planning and cooperation. The experience gained from this review will subsequently be used in similar reviews of the existing European 8-10m telescopes, and later of the radio telescopes as well. 
The impending decision points on some of the large projects (ELTs, SKA, ...) will provide a good opportunity to re-think the organization of the smaller facilities as well. Hopefully, the funding agencies will then follow up this review with far-sighted decisions, which will enable us to undertake the large future programmes described in the Science Vision. In a world of finite resources, such a gain will come at the expense of some of the traditional P.I.-driven science, so interaction with the community during the review process will again be crucial.

The ASTRONET review is not conceived in an atmosphere of isolation: Its goals are similar to the ReSTAR and ALTAIR reviews in the USA, and contacts are maintained to identify potential areas of synergy. Having clear overviews of the aims and capabilities of both sides will be useful in the next step, optimising the overall scientific returns on a global basis. Obvious opportunities for cooperation are:

Science cases for individual $2-4 \mathrm{~m}$ telescopes,

Hands-on training of the next generation of astronomer/engineers,

Complementarity in instrumentation and sky coverage,

Instrument/detector/software development,

Standardised telescope performance criteria,

Time domain astronomy and distributed facilities, and

Optimising common use of observatory sites and coordinate operations.

Recent developments and funding decisions affecting our present complement of mediumsize telescopes have not conformed particularly accurately to this ideal. But if astronomers cannot lead the way to effective global scientific cooperation, who can?

\section{References}

Bode, M. F., Cruz, M. D., \& Molster (Eds.) 2008, An Infrastructure Roadmap for European Astronomy, ASTRONET

de Zeeuw, P. T. \& Molster, F. J. (Eds.) 2007, A Science Vision for European Astronomy, ASTRONET

ALTAIR: http://www.noao.edu/system/altair/

ASPERA: http://www.aspera-eu.org/

ASTRONET: http://www.astronet-eu.org

ESFRI: http://cordis.europa.eu/esfri/

EuroPlaNet: http://www.europlanet-eu.org

Nordström, B., Mayor, M., \& Andersen, J., et al. 2004, A\&\&A 418, 989

OECD Global Science Forum: http://www.oecd.org/

RadioNet: http://www.radionet-eu.org/

ReSTAR: http://www.noao.edu/system/restar/ 